\title{
Heating Effects from Driven Transverse and Alfvén Waves in Coronal Loops
}

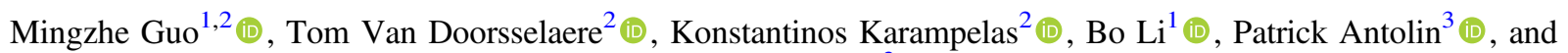 \\ Ineke De Moortel $^{3}$ (D) \\ ${ }^{1}$ Institute of Space Sciences, Shandong University, Weihai 264209, People's Republic of China; \\ ${ }^{2}$ Centre for mathematical Plasma Astrophysics, Department of Mathematics, KU Leuven, B-3001 Leuven, Belgium; mingzhe.guo@kuleuven.be \\ ${ }^{3}$ School of Mathematics and Statistics, University of St. Andrews, St. Andrews, Fife KY16 9SS, UK \\ Received 2018 September 20; revised 2018 November 15; accepted 2018 November 16; published 2019 January 8
}

\begin{abstract}
Recent numerical studies revealed that transverse motions of coronal loops can induce the Kelvin-Helmholtz instability (KHI). This process could be important in coronal heating because it leads to dissipation of energy at small spatial scale plasma interactions. Meanwhile, small-amplitude decayless oscillations in coronal loops have been discovered recently in observations of $S D O /$ AIA. We model such oscillations in coronal loops and study wave heating effects, considering a kink and Alfvén driver separately and a mixed driver at the bottom of flux tubes. Both the transverse and Alfvén oscillations can lead to the KHI. Meanwhile, the Alfvén oscillations established in loops will experience phase mixing. Both processes will generate small spatial scale structures, which can help the dissipation of wave energy. Indeed, we observe the increase of internal energy and temperature in loop regions. The heating is more pronounced for the simulation containing the mixed kink and Alfvén driver. This means that the mixed wave modes can lead to a more efficient energy dissipation in the turbulent state of the plasma and that the KHI eddies act as an agent to dissipate energy in other wave modes. Furthermore, we also obtained forward-modeling results using the FoMo code. We obtained forward models that are very similar to the observations of decayless oscillations. Due to the limited resolution of instruments, neither Alfvén modes nor the fine structures are observable. Therefore, this numerical study shows that Alfvén modes probably can coexist with kink modes, leading to enhanced heating.
\end{abstract}

Key words: magnetohydrodynamics (MHD) - Sun: corona - Sun: magnetic fields - waves

\section{Introduction}

A rich variety of MHD oscillations and waves have been observed in the highly structured solar atmosphere (for recent reviews, see, e.g., Nakariakov \& Verwichte 2005; De Moortel \& Nakariakov 2012; Jess et al. 2015; Nakariakov et al. 2016). They may play an important role in coronal heating because of their capability of carrying energy (e.g., Taroyan \& Erdélyi 2009; Parnell \& De Moortel 2012). In fact, analytical studies to reveal the various wave properties in magnetic structures date back to the 1970s (Zajtsev \& Stepanov 1975; Wentzel 1979). In 1999, the first imaging observation of kink waves in active region loops was obtained by the Transition Region and Coronal Explorer (Aschwanden et al. 1999; Nakariakov et al. 1999; Schrijver et al. 1999). Since then, a large number of transverse waves have been discovered in the solar atmosphere by modern instruments (e.g., Nakariakov \& Verwichte 2005; Verwichte et al. 2005; Aschwanden 2006; Tomczyk \& McIntosh 2009), not only in coronal loops (see Ruderman \& Erdélyi 2009, for a recent review), but in chromospheric spicules and mottles (e.g., De Pontieu et al. 2007; He et al. 2009; Kuridze et al. 2012, 2013; Morton et al. 2012), large prominences (e.g., Arregui et al. 2012), polar plumes (e.g., Gupta et al. 2010), and coronal streamers (e.g., Chen et al. 2010, 2011; Kwon et al. 2013).

The observed large-amplitude transverse oscillations generally undergo rapid damping in a couple of periods (Nakariakov et al. 1999; Aschwanden et al. 2002; Goddard et al. 2016). Such damping is usually attributed to resonant absorption (Hollweg \& Yang 1988; Goossens et al. 1992, 2002) or phase mixing (Soler \& Terradas 2015). The kink oscillations transfer into local Alfvén modes when the kink frequency matches the local Alfvén frequency; therefore, the transverse motion has an apparent decay. This process is usually expected to happen in an inhomogeneous layer near the loop boundary. On the other hand, Soler \& Terradas (2015) considered the phase mixing process simultaneously, which is responsible for the wave energy transfer from large spatial scale structures to small-scale plasma interactions. The real dissipation of wave energy at such small structures relies on resistivity and viscosity (Ofman et al. 1994, 1998).

Recent simulations of transverse waves in coronal loops revealed that the Kelvin-Helmholtz instability (KHI) occurs near the boundary of loops (Terradas et al. 2008; Antolin et al. 2014, 2017; Magyar et al. 2015; Howson et al. 2017a, 2017b; Karampelas et al. 2017). The instability is generated as a result of the strong shear motions near the loop edges. Meanwhile, the generation of azimuthal Alfvén waves at resonant layers increases the velocity shear with the external plasma, which can also enhance the instability and make the systems more unstable. Antolin et al. (2014) revealed that even a smallamplitude $\left(\sim 3 \mathrm{~km} \mathrm{~s}^{-1}\right)$ kink oscillation can lead to such an instability. The importance of this transverse-wave-induced Kelvin-Helmholtz (TWIKH) instability (Antolin et al. 2014, 2017) is that it generates turbulent small structures. This makes the wave energy dissipate much more easily in the small-scale structures in the presence of transport coefficients or kinetic effects. This is probably a crucial process for coronal heating (Howson et al. 2017a; Karampelas et al. 2017).

Due to their incompressibility, Alfvén waves are not easily detected by imaging instruments in the solar atmosphere. Their torsional motions would cause spectral line broadening, making them detectable to spectrographs (Zaqarashvili 2003). Jess et al. (2009) reported the torsional Alfvén waves in the chromosphere, using the $\mathrm{H} \alpha$ line in the Solar Optical universal Polarimeter of Spitzer Space Telescope. However, the corresponding coronal observations remain unclear. 
Theoretically, Alfvén waves can be easily generated from the lower atmosphere (Muller et al. 1994; Beliën et al. 1999). Vranjes et al. (2008) claimed that the wave energy flux through the photosphere becomes orders of magnitude smaller when considering the effects of partial ionization and collisions. However, the fast waves transfer their energy to up-going Alfvén waves in the conversion region. The process is analogous to the resonant absorption mentioned above, making the Alfvén flux increase significantly in the lower atmosphere (Khomenko \& Cally 2012; Cally \& Moradi 2013; Grant et al. 2018). Similar to kink waves, the energy dissipation is an important issue. Heyvaerts \& Priest (1983) claimed that phase mixing occurs between different magnetic surfaces when Alfvén waves propagate in the nonuniform magnetic structures. Recent work by Pagano et al. (2018) found that heating from phase mixing of Alfvén modes in coronal loops with multiharmonic oscillations is small. However, the KHI can be induced for standing modes eventually owing to the strong, localized velocity shear. Such small turbulent structures induced through the instability can help wave energy dissipate more easily.

Recently, low-amplitude decayless transverse oscillations have been detected (Anfinogentov et al. 2013, 2015; Nisticò et al. 2013). Imaging observations (such as SDO/AIA) revealed that this kind of apparently undamped oscillation is a common phenomenon in coronal loops with small average amplitudes. Nisticò et al. (2013) initially interpreted such undamped regimes as the response of loops to external continuous drivers. Antolin et al. (2016) explained such oscillations as combined effects of periodic brightening of TWIKH rolls and the limited resolution of instruments. Nakariakov et al. (2016) proposed that these decayless oscillations were caused by interaction of loops with quasisteady flows as self-oscillations. Very recently, Karampelas et al. (2017) and Karampelas \& Van Doorsselaere (2018) simulated such decayless transverse oscillations as coronal loops driven by transverse motions.

In this article, we aim to simulate such driven oscillations and study the heating effects, considering a mixed kink and Alfvén driver at one footpoint of a loop. Matsumoto \& Shibata (2010) claimed that turbulent photospheric motions can be observed by Hinode/SOT; therefore, it is reasonable to expect mixed motions at the footpoints of loops. The mixed processes of KHI, resonant absorption, and phase mixing will greatly influence the heating effects. Meanwhile, the decayless oscillations are ubiquitous in coronal loops, so it is worthwhile to reveal their relation to coronal heating. For comparison, pure Alfvén and kink driver models are also considered. This paper is organized as follows. Section 2 presents our basic setup of numerical models. Apparent dynamics of the loops are presented in Section 3. In Section 4, we analyze the energy variations of the three models to examine the heating effects. Forward modeling is performed in order to compare to real observations in Section 5. Finally, Section 6 closes this paper with discussions and conclusions.

\section{Numerical Models}

\subsection{Equilibrium and Drivers}

We consider three 3D numerical models in our simulations. They are all based on the same straight density-enhanced magnetic tube, which is embedded in a uniform background
Table 1

Parameters Used in Simulations

\begin{tabular}{lc}
\hline \hline Parameters & Values \\
\hline Loop length $L(\mathrm{Mm})$ & 150 \\
Loop radius $R(\mathrm{Mm})$ & 1 \\
Internal density $\rho_{\mathrm{i}}\left(\mathrm{g} \mathrm{cm}^{-3}\right)$ & $2.5 \times 10^{-15}$ \\
Density ratio $\rho_{\mathrm{i}} / \rho_{\mathrm{e}}$ & 3 \\
Temperature $T(\mathrm{MK})$ & 1 \\
Magnetic field $B_{\mathrm{i}}(\mathrm{G})$ & 50 \\
\hline
\end{tabular}

plasma. We aim to model a coronal loop with a uniform magnetic field directed along the $z$-direction. Similar models have been used in previous works (e.g., Antolin et al. 2014, 2017; Magyar et al. 2015; Karampelas et al. 2017; Karampelas $\&$ Van Doorsselaere 2018). The loop has an initial density ratio of $\rho_{\mathrm{i}} / \rho_{\mathrm{e}}=3$ (index i [e] denotes internal [external] values), and we consider a density profile given by

$$
\begin{gathered}
\rho(x, y)=\rho_{\mathrm{e}}+\left(\rho_{\mathrm{i}}-\rho_{\mathrm{e}}\right) \phi(x, y), \\
\phi(x, y)=\frac{1}{2}\left\{1-\tanh \left[b\left(\sqrt{x^{2}+y^{2}} / R-1\right)\right]\right\},
\end{gathered}
$$

where, $x, y$ denote the coordinates in the plane perpendicular to the direction of the loop, which is fixed as the $z$-direction. $b$ sets the width of the boundary layer. We choose $b=8$, which gives the width of the inhomogeneous layer $l \approx 0.4 R$, corresponding to a typical value estimated in coronal loops (Goossens et al. 2002). The initial parameters of the loop are shown in Table 1 . The loop length $(L=150 \mathrm{Mm})$ and radius $(R=1 \mathrm{Mm})$ are chosen within the range of observations (Nakariakov et al. 1999; Aschwanden et al. 2002). The density ratio here is chosen according to the estimated value in Aschwanden et al. (2003).

We consider a uniform temperature loop $\left(T_{\mathrm{i}}=T_{\mathrm{e}}=1 \mathrm{MK}\right)$, so the average temperature increase due to the mixing between the colder tube and hotter background corona is avoided (Karampelas et al. 2017). Therefore, it will be easier to identify the true wave heating effects. To maintain the magnetostatic pressure balance, the magnetic field has a slight variation from internal $B_{\mathrm{i}}=50 \mathrm{G}$ to external $B_{\mathrm{e}}=50.07 \mathrm{G}$.

The magnetic field $(50 \mathrm{G})$ here is larger than in previous models (e.g., Antolin et al. 2014, 2017; Karampelas et al. 2017) and observations (e.g., Nakariakov \& Ofman 2001; Van Doorsselaere et al. 2007, 2008; Jess et al. 2016). In this case, the energy input into the models is increased in order to obtain more noticeable heating effects.

In order to investigate the heating effects of different wave modes, we employ three models with the same initial parameters in Table 1, but with different drivers on the bottom footpoint $(z=0)$. The first driver is a continuous, monoperiodic "dipole-like" driver, which is similar to Pascoe et al. (2010) and Karampelas et al. (2017). The time-dependent velocity inside the loop $(r<R)$ is

$$
\boldsymbol{v}_{\mathrm{i}}=v_{0}\left[\sin \left(\frac{2 \pi t}{P_{\mathrm{k}}}\right), 0,0\right],
$$

where $v_{0}=2 \mathrm{~km} \mathrm{~s}^{-1}$ is the amplitude of the velocity. The period of the driver $P_{\mathrm{k}}=87 \mathrm{~s}$, which corresponds to the predicted value for the fundamental kink mode (Edwin \& Roberts 1983). The 


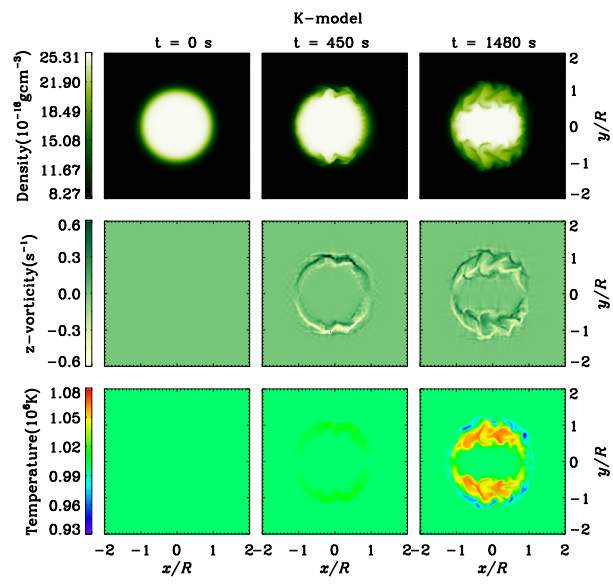

(a)
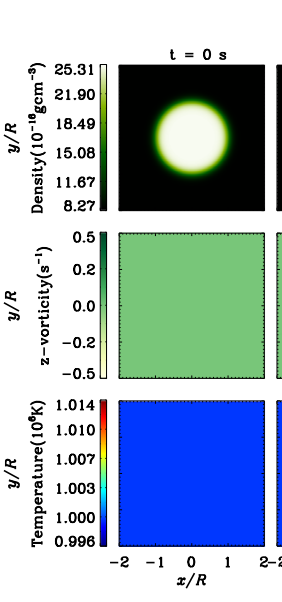

A-model
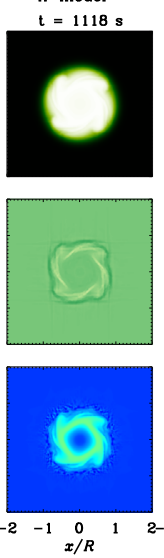

(b)

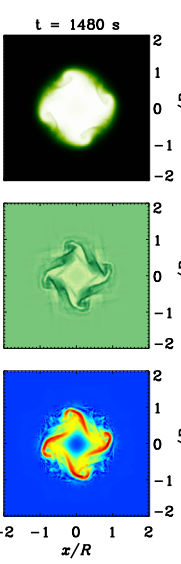

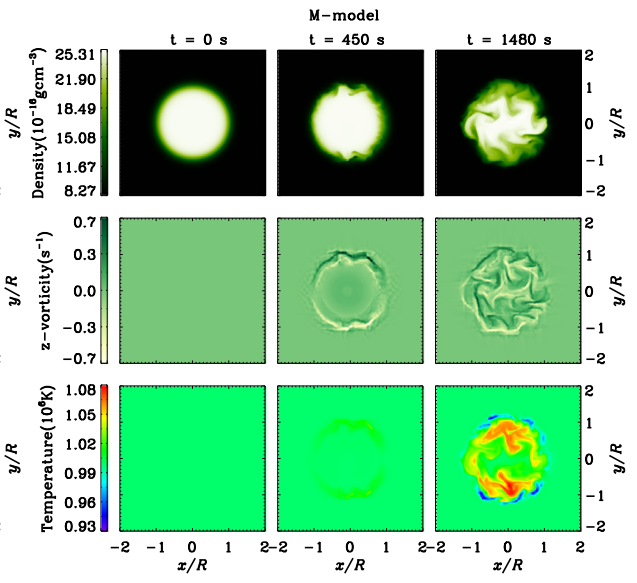

(c)

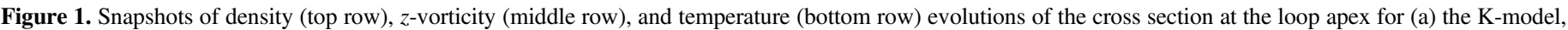
(b) the A-model, and (c) the M-model.

spatial dependence of the driver velocity outside the loop has the form

$$
v_{\mathrm{e}}=v_{0} R^{2} \sin \left(\frac{2 \pi t}{P_{\mathrm{k}}}\right)\left[\frac{x^{2}-y^{2}}{\left(x^{2}+y^{2}\right)^{2}}, \frac{2 x y}{\left(x^{2}+y^{2}\right)^{2}}, 0\right] .
$$

We also use a transition layer between these two regions to avoid the numerical problems, as Pascoe et al. (2010) and Karampelas et al. (2017) did. The profile is similar to the density profile given by Equations (1) and (2).

The second driver is a broadband time-dependent torsional motion, mimicking Alfvén oscillations inside a loop. The torsional driver is inspired by the one used in Beliën et al. (1999). To launch this driver, $v_{\theta}$ is described as

$$
v_{\theta}=v_{0} \sin \left(\frac{2 \pi t}{P_{\mathrm{A}}(r)}\right)\left\{\begin{array}{ll}
\left(\frac{2 r}{R}\right)^{2}\left(\frac{2 r}{R}-2\right)^{2}, & r / R \leqslant 1 \\
0, & r / R>1
\end{array},\right.
$$

where $v_{0}$ keeps the same value as that of the kink driver. The period $P_{\mathrm{A}}$ is a function of radial distance because we have a nonuniform transverse density distribution. It is given by $P_{\mathrm{A}}(r)=2 L / v_{\mathrm{A}}(r)=2 L \sqrt{\mu_{0} \rho(r)} / B(r)$, varying from its internal value of $106 \mathrm{~s}$ to the loop boundary $(r=R)$ value of $87 \mathrm{~s}$. Using these periods, we can establish Alfvén oscillations in the uniform region $(r<0.8 R)$ and the inhomogeneous region $(0.8 R \leqslant r \leqslant R)$ with the corresponding periods on the different magnetic surfaces.

Finally, the third driver is a mixed Alfvén and kink driver. We consider both transverse velocity (given by Equations (3) and (4)) and torsional motions (given by Equation (5)) simultaneously during the entire simulation. Therefore, the energy provided by the mixed driver, i.e., input energy, is at the same level as the sum of the other two drivers.

For simplicity, hereafter we name the kink driver model the "K-model," the Alfvén driver model the "A-model," and the mixed driver model the "M-model." In our K-model and M-model, the drivers follow the motions of loops, making sure that the internal loop regions will always have a uniform velocity.

\subsection{Numerical Setup}

To solve the 3D time-dependent MHD equations, we use the PLUTO code (Mignone et al. 2007). A second-order parabolic spatial scheme is used for integration, and the numerical fluxes are computed by a Roe Riemann solver. Meanwhile, a third-order Runge-Kutta algorithm is used to advance the solution to the next time level. The simulation domain is $[-8,8] \mathrm{Mm} \times[-8,8] \mathrm{Mm} \times[0,150] \mathrm{Mm}$. To resolve the motions of the drivers near the footpoints, we adopt a stretched mesh with 5 cells from 0 to $R$ and a uniform grid of 95 points from $R$ to $L$ in the $z$-direction. For the $x$ - and $y$-directions, 256 nonuniformly spaced cells are adopted. The resolution is up to $20 \mathrm{~km}$ in the region of $|x, y| \leqslant 2 \mathrm{Mm}$. The following simulations show that this resolution is high enough to observe small structures induced by waves and instabilities.

In order to establish standing waves in loops, we fix the velocities at $z=L$ to be zero to mimic loops anchored in the photosphere. The other variables there are set to obey Neumann-type (zero-gradient) conditions. The $z$-component velocities at the bottom footpoint $(z=0)$ are antisymmetric, and $v_{x}, v_{y}$ are described by the drivers. All the lateral boundaries are set to be outflow conditions.

\section{General Numerical Results}

We ran simulations until $t=1500 \mathrm{~s}$ for all three models, corresponding to roughly $14-17$ periods. The maximum displacements the loops experienced are less than $1 \mathrm{Mm}$, to allow us to concentrate on the subdomain of $|x, y| \leqslant 2 \mathrm{Mm}, 0 \leqslant z \leqslant 150 \mathrm{Mm}$, which is the domain with the highest resolution in the $x, y$ directions.

\subsection{KHI Eddies, Resonant Absorption, and Phase Mixing}

The simulation results show that the loops quickly form driven standing waves in the three models, namely, standing kink (Alfvén) waves in the K-model (A-model) and mixed (both standing kink and Alfvén) modes in the M-model. As in previous studies, the generation of KHI can also be seen in our $\mathrm{K}$-model, as is shown in Figure 1(a). The KHI develops near $|y|=R$, inducing the so-called TWIKH rolls (Antolin et al. 2014, 2017). Figure 1(b) shows that axisymmetric vortices occur around the loop boundary in the A-model. This means 


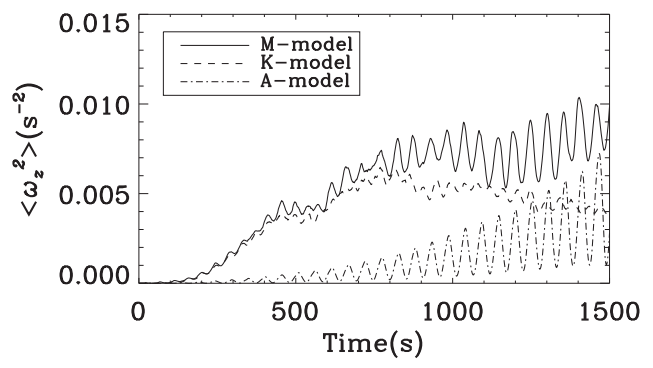

Figure 2. Time evolution of the averaged square $z$-vorticity $\left(\omega_{z}^{2}\right)$ for the M-model (solid line), K-model (dashed line), and A-model (dot-dashed line). The quantities are averaged over the region of $|x, y| \leqslant 2 R$ at the loop apex.

that the Alfvén oscillations in a nonuniform layer can also induce the instability, which corresponds to the prediction of Heyvaerts \& Priest (1983). We can observe four clear eddies around $|y|=R$ at $t=450 \mathrm{~s}$ in the K-model. Actually, there are still four small eddies beside the clear ones around the loop boundary, which can be observed in the later instant $(t=1480 \mathrm{~s})$. This means that the initial unstable mode in the $\mathrm{K}$-model has a wavenumber of $m=8$. In the A-model, four eddies start to occur at $t=1118 \mathrm{~s}$, indicating that the wavenumber of the initial unstable mode is $m=4$.

The results of the M-model are shown in Figure 1(c). It is almost the same snapshot as in the K-model at $t=450 \mathrm{~s}$, indicating that the torsional motions inside the loop have little influence on the instability near the loop boundary initially. When the instability induced from the torsional waves develops, the loop is deformed and the eddies extend from the boundary to almost the whole region in the M-model, as is indicated by the $z$-vorticity in the middle panel of Figure 1. Note that the results here are attributed to not only the effect of mixed motions but also a higher energy input in the M-model than in the other two models.

We also plot the temperature evolution of the apex in the bottom row of Figure 1. The temperature increases at the locations where small eddies develop for all three models. Meanwhile, we can also observe a temperature decrease around the boundary edges. The fluctuations of the temperature probably do not mean that the heating indeed happens at those small spatial scale structures. This property is explained as adiabatic heating (cooling) rather than real dissipation (Magyar et al. 2015; Antolin et al. 2017, 2018; Karampelas et al. 2017). It should be noted that the temperature increase in the A-model is smaller than in the other two models. This means that the Alfvén modes do not produce so many small eddies to deform the loop; therefore, the density has a smaller change, leading to a smaller temperature increase.

To quantify the turbulent level in our models, we examine the averaged square $z$-vorticity $\left(\omega_{z}^{2}\right)$ at the loop apex, which is shown in Figure 2. The $\omega_{z}^{2}$ in the M-model is the largest, indicating that the instability in this model is the strongest. However, the amplitude increase of $\omega_{z}^{2}$ in the A-model does not mean that more eddies are generated in this model. It is mainly due to the increasing torsional motions at the loop apex.

Alfvén modes converted from kink oscillations through resonant absorption can be easily seen near the loop boundaries (Hollweg \& Yang 1988; Goossens et al. 1992, 2002). Figures 3 (a) and (c) show the velocity spikes near $|y|=R$ in the $\mathrm{K}$-model and the M-model, respectively, at $t=255 \mathrm{~s}$. The spikes are the Alfvén modes converted from kink oscillations. We do not find the Alfvén modes at the same locations in the
A-model in Figure 3(b), because no kink oscillations appear in this model. The crests near $y=-0.5 R$ and the troughs near $y=0.5 R$ in the A-model (Figure 3(b)) and the M-model (Figure 3(c)) are the Alfvén oscillations coming from the drivers. It should be noted that in the M-model the Alfvén oscillations inside the loops can mix with the Alfvén modes in the nonuniform layer owing to their different periods, inducing the KHI. Hence, small structures can be seen near $y=-0.8 R$ in Figure 3(c).

The Alfvén oscillations with different frequencies can have phase mixing after a number of periods (Heyvaerts \& Priest 1983). However, the scales of phase mixing eddies will decrease over time, since phase mixing will generate larger gradients and smaller-scale structures. According to Mann et al. (1995; see also Kaneko et al. 2015; Raes et al. 2017), the finestscale structures are governed by the phase mixing length

$$
L_{\mathrm{ph}}=\frac{2 L}{t\left(v_{\mathrm{Ae}}-v_{\mathrm{Ai}}\right) / l} \text {. }
$$

For a very late instant $t=1480 \mathrm{~s}$, the phase mixing length is $L_{\mathrm{ph}}=0.039 \mathrm{Mm}$, which is already very close to our numerical resolution. Hence, we cannot clearly observe such small structures any more. Besides, the onset of the instability can also make the identification of the phase mixing fine structures become ambiguous.

More eddies occur in the M-model, indicating that the mixed torsional and transverse motions deform the loop efficiently. Meanwhile, considering the small structures induced by phase mixing, we find that the mixed modes are more efficient in generating such small spatial scale structures. Therefore, they are also likely to dissipate energy into heating more efficiently.

\subsection{The Saturation of Oscillations}

Once we set up fundamental kink oscillations in loops, the direct approach is to check the displacements or velocities at the apex, which is the location of the antinode of transverse motions. However, because of the deformation of loops, the displacement of the apex cannot reveal the true oscillation properties any more. Although the deformations of our loops are not as strong as those of Karampelas \& Van Doorsselaere (2018) owing to our smaller period and larger magnetic field, to avoid the influence of the deformation, we choose the perturbations of the transverse magnetic field at the footpoint to examine the oscillation properties. For fundamental oscillations in loops, the perturbations of the transverse magnetic field will have its maximum values at the footpoints. Figure 4 shows the transverse perturbations of the magnetic field at the point $[0.5 R$, $0,0]$ in the three models. The specific point here is fixed at the bottom plane, so it is not advected following the drivers. The maximum displacement of the central loop region in this plane is about $27 \mathrm{~km}$, which is close to our resolution of $20 \mathrm{~km}$. Hence, considering a fixed point does not significantly influence the results.

Figure 4(a) shows the profiles of $b_{x}$ in the M-model and the K-model. Since the Alfvén motions do not have an $x$-component inside the loops, the $b_{x}$ here mainly represents the kink motions. The amplitudes of $b_{x}$ in the two models are identical before $t=1100 \mathrm{~s}$, showing that the kink oscillations are formed in both models, and they quickly achieve the same saturation after about three periods owing to resonant absorption. However, the Alfvén modes need a longer time to 

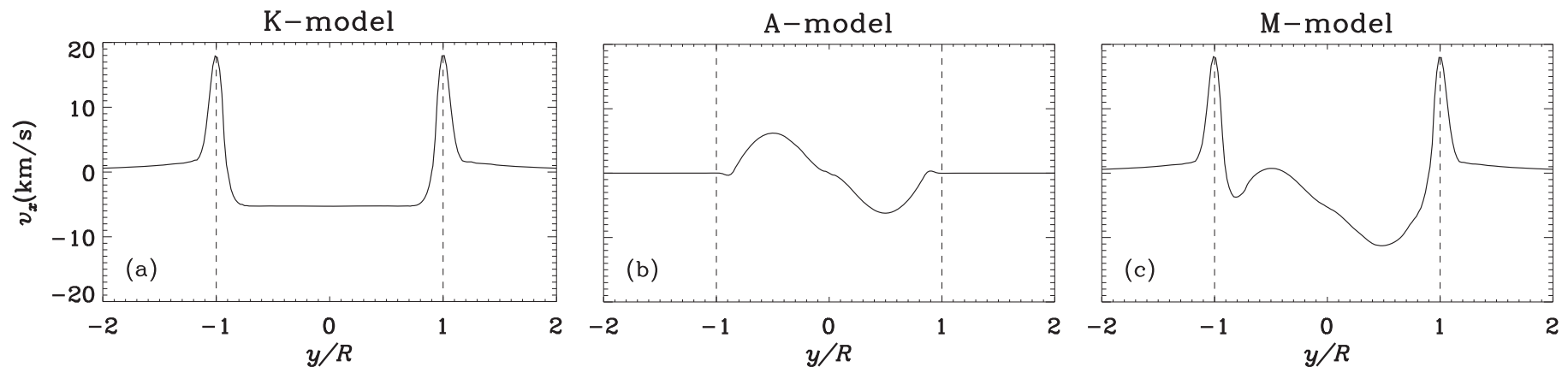

Figure 3. The $v_{x}$ profile along the $y$-direction at $x=0$ and at the apex of the loops for (a) the K-model, (b) the A-model, and (c) the M-model at $t=255 \mathrm{~s}$. Vertical dashed lines represent the locations of loop boundaries.
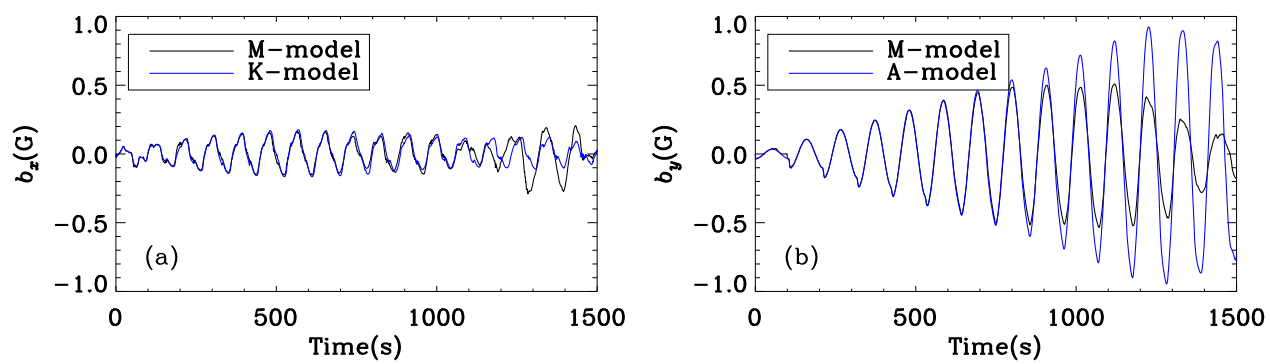

Figure 4. Transverse magnetic field perturbations at $[0.5 R, 0,0]$ in the three models. The point is a fixed one that is not advected according to the drivers. The left panel shows the $b_{x}$ evolution for the M-model (black line) and the K-model (blue line). The right panel shows the $b_{y}$ evolution for the M-model (black line) and the A-model (blue line).
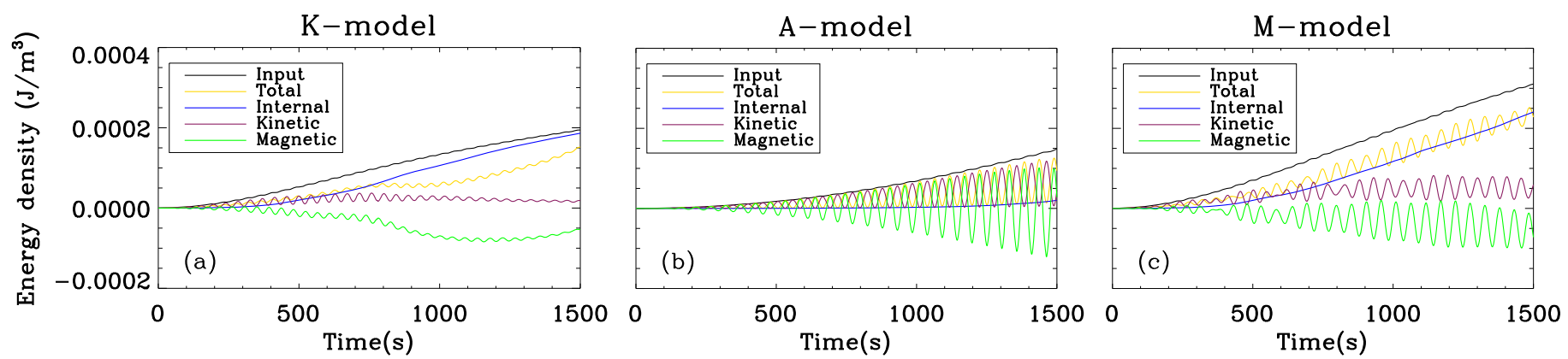

Figure 5. Volume-averaged energy density variations relative to the initial state for (a) the K-model, (b) the A-model, and (c) the M-model. The energy densities are volume averaged over the region of $|x, y| \leqslant 2 R, 0 \leqslant y \leqslant L$. Different colors represent different kinds of energy density variations. Note that the total energy density means the sum of internal, kinetic, and magnetic energy density.

saturate, leading to larger saturation values, as is shown in Figure 4(b). The amplitudes of $b_{y}$ in the M-model and the A-model are identical before $t=800 \mathrm{~s}$. Then, the saturation comes after that in the M-model, while it saturates after about $t=1200 \mathrm{~s}$ in the A-model. It should be noted that the amplitude of $b_{x}$ in the M-model increases after about $t=1200 \mathrm{~s}$, whereas the amplitude of $b_{y}$ decreases after about $t=1200 \mathrm{~s}$. This is because the point chosen here is very close to the edge of an eddy, which makes the magnetic field vector component in the bottom plane deflect to the $x$-direction.

\section{Energetics}

To understand the energy transfer in the systems, we study the time evolution of different kinds of energy. In the following parts, we will analyze volume-averaged values in the subregion of $|x| \leqslant 2 R,|y| \leqslant 2 R, 0 \leqslant y \leqslant L$. The input energy, namely, the Poynting flux provided by the driver, is calculated by

$$
S(t)=-\frac{1}{V} \int_{0}^{t} \int_{A} \boldsymbol{S} \cdot d \boldsymbol{A} d t^{\prime}
$$

following the definition in Beliën et al. (1999). Here, $\boldsymbol{S}$ is the Poynting flux, $\boldsymbol{A}$ is the normal surface vector of the bottom plane, and $V$ is the total volume of the subregion.

Since all the variations are averaged in the same subvolume, we will discuss energy instead of energy density in the following. In Figure 5, the input energy for each model is approximately divided between the internal energy and the kinetic energy. In the K-model, a quick saturation in the kinetic energy is achieved, with a slight decrease after 10 periods. This is because the collective transverse oscillation transfers into the local turbulent motions near the loop boundary, and then the TWIKH rolls break up into smaller and smaller structures. Meanwhile, considering the extension of the nonuniform layer (Karampelas et al. 2017), these fine structures spread over a larger region, causing the decrease of the averaged velocity. Similar reduction in the vorticity of the K-model can also be observed in Figure 2. Because of the decrease of the magnetic field perturbation in the bottom plane, the input energy experiences a slower increase in the later periods in the $\mathrm{K}$-model. In the A-model and the M-model, both the kinetic 

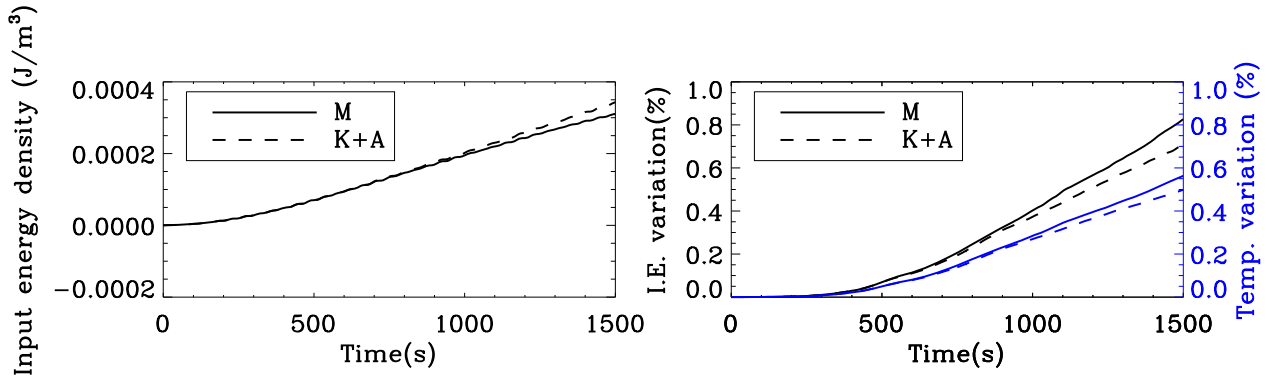

Figure 6. Left: volume-averaged input energy density variations. Right: percentages of volume-averaged internal energy (black) and temperature (blue) variations. Solid lines represent the M-model; dashed lines represent the sum of the K-model and the A-model. The quantities are volume averaged over the region of $|x, y| \leqslant 2 R, 0 \leqslant y \leqslant L$.

energy and the magnetic energy have larger relative amplitudes, indicating later saturations. Note that in the M-model beatings can be seen in the amplitudes of the kinetic energy and the magnetic energy owing to the period mismatch between the transverse and torsional waves.

The drop in the magnetic energy, namely, the difference between the input energy and the total energy, is caused mainly by the Poynting flux through our open lateral boundaries. Meanwhile, the other part can be attributed to the energy transfer from the magnetic energy to the internal energy due to the effect of numerical resistivity. This is similar to the results of Karampelas et al. (2017). We also notice a small rise near the end of our simulation for magnetic energy in the K-model, which is also mentioned by Karampelas et al. (2017). This is due to the continuous energy input of the driver.

The input energy is almost at the same level in the K-model and the A-model; however, the internal energy increase in the A-model is much smaller than in the K-model. As is mentioned in Section 3.1, the pure torsional motions produce fewer eddies in the A-model. Therefore, the wave energy is less dissipated, showing a very weak heating here.

In Figure 5(c), the increased input energy in the M-model becomes approximately proportional to time after about $t=500 \mathrm{~s}$. We estimate the energy flux $E=\Delta S(t) V / \Delta t A \sim 36.5 \mathrm{~W} \mathrm{~m}^{-2}$, choosing a period from 700 to $1200 \mathrm{~s}$. This energy flux seems to get close to balancing the radiative energy losses of the quiet corona, $\sim 100 \mathrm{~W} \mathrm{~m}^{-2}$ (Withbroe \& Noyes 1977; Tomczyk et al. 2007). Furthermore, it should be noted that the input energy flux would increase for a larger input velocity. If we consider a larger-amplitude driver, for example, $4 \mathrm{~km} \mathrm{~s}^{-1}$, the input kinetic energy would become four times larger, which would be enough to heat at least the quiet corona. Such a larger amplitude could be representative of driving velocities in, e.g., the chromosphere.

To clearly compare the variations of internal energy and temperature in all three models, we examine the percentages of volume-averaged values. Before that, we compare the input energy in the M-model and the sum of the other two models, as is shown in the left panel of Figure 6. They are identical before $t=1000 \mathrm{~s}$, and then the input energy in the M-model becomes smaller than the sum of the other two models. This is due to the decrease of the magnetic field perturbations near the footpoint in the M-model. The right panel of Figure 6 shows that the relative variations of internal energy and temperature monotonically increase over time. For the M-model, the relative variation of the internal energy increases to $0.83 \%$ at the end of the simulation $(t=1500 \mathrm{~s})$. Meanwhile, it increases to $0.71 \%$ for the sum of the other two models. Similarly, the relative variation of the temperature increases to $0.56 \%$ at the end of the simulation for the M-model and to $0.49 \%$ for the sum of the other two models. Although the input energy is even smaller in the later periods of the simulation, both the internal energy and the temperature still get larger increases in the M-model. This means that the mixed modes in the M-model can indeed have enhanced heating due to a more efficient dissipation than the other two models combined. As such, the KHI rolls act as a catalyst to more efficiently dissipate the energy in other wave modes.

\section{Observable Properties}

To obtain observable signals and compare to real observations, we forward-modeled the numerical simulations using the FoMo code (Van Doorsselaere et al. 2016). The Fe IX $171 \AA$ emission line is chosen since it is sensitive to the temperature of the models here.

Figure 7(a) shows the results for the K-model, where the left column shows the time-distance diagram of the normalized intensity in the Fe IX $171 \AA$ line at the loop apex with a line-of-sight (LOS) angle of $45^{\circ}$ (LOS angle of $0^{\circ}$ along the $y$-direction) within the plane perpendicular to the loop axis. The upper image is obtained with the full numerical resolution, and the fine strand-like structures can be clearly seen owing to the instability after about $450 \mathrm{~s}$, which can also be seen in impulsively excited loops (Antolin et al. 2014, 2016, 2017). Similar to the results of Antolin et al. (2016), the periodic increase of intensity after about $500 \mathrm{~s}$ around the boundary is caused by the TWIKH rolls. The intensity increase is more apparent here owing to the continuous energy increase in our model. The fine structures dim after about $1000 \mathrm{~s}$, as the eddies break into much smaller ones, so the smaller structures can no longer be seen. To compare to the observations of decayless oscillations in coronal loops, we degrade the original spatial resolution to that of a given imaging instrument, namely, $S D O /$ AIA here. The result is shown on the bottom left of Figure 7(a). It is very similar to the observations reported by Anfinogentov et al. (2013, 2015), meaning that our simulation agrees with a decayless transverse oscillation. The same decayless oscillations can also be seen in the models of Antolin et al. (2016) with a coarse instrument resolution. The middle column of Figure 7(a) shows the Doppler velocities in the same emission line and an LOS angle of $45^{\circ}$. Staggered blue- and redshifts appear, showing a series of "bow-like" shapes. It should be noted that they are similar to the results of Antolin et al. (2017), the moving of crests opposite to the loop core and their troughs move in the same phase as the loop core. Smaller structures can now be seen after about $1000 \mathrm{~s}$, which agrees with the above statements. To compare with a real instrument, we also degrade the original numerical spatial resolution to $3^{\prime \prime}$. We 
(a) K-model
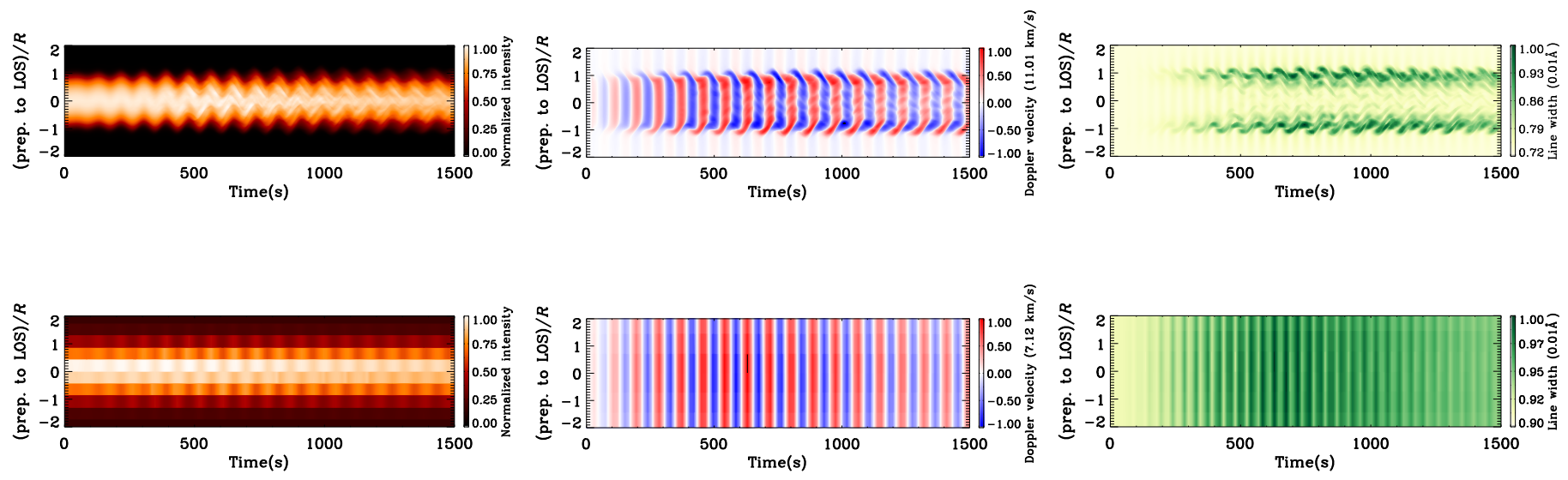

(b) A-model
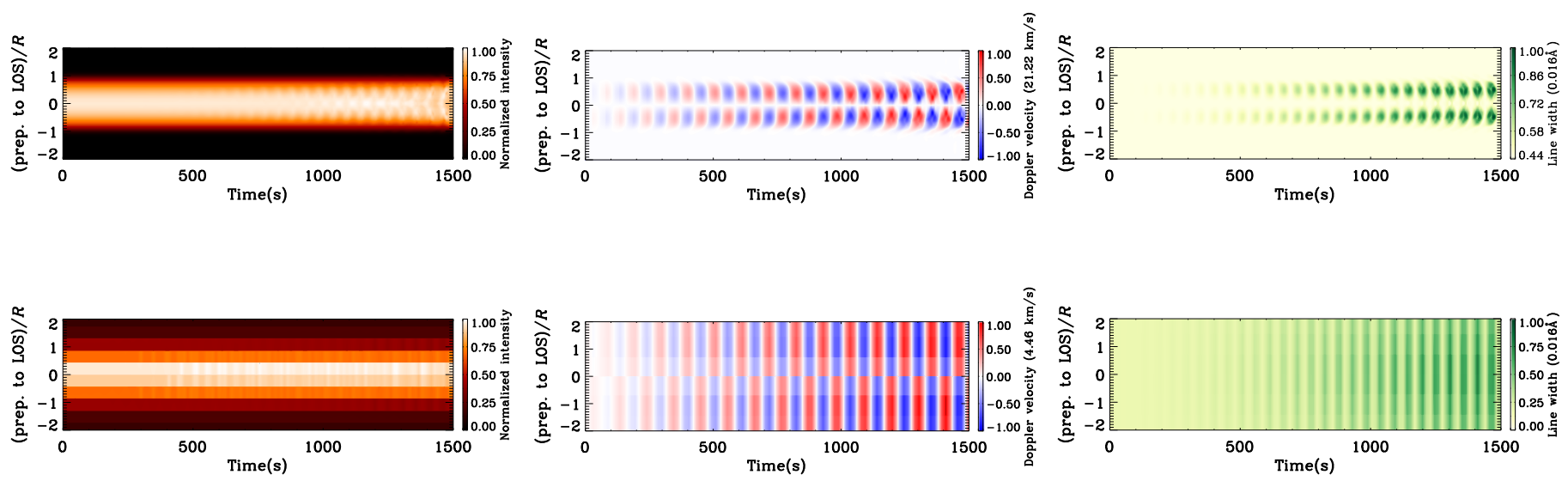

(c) M-model
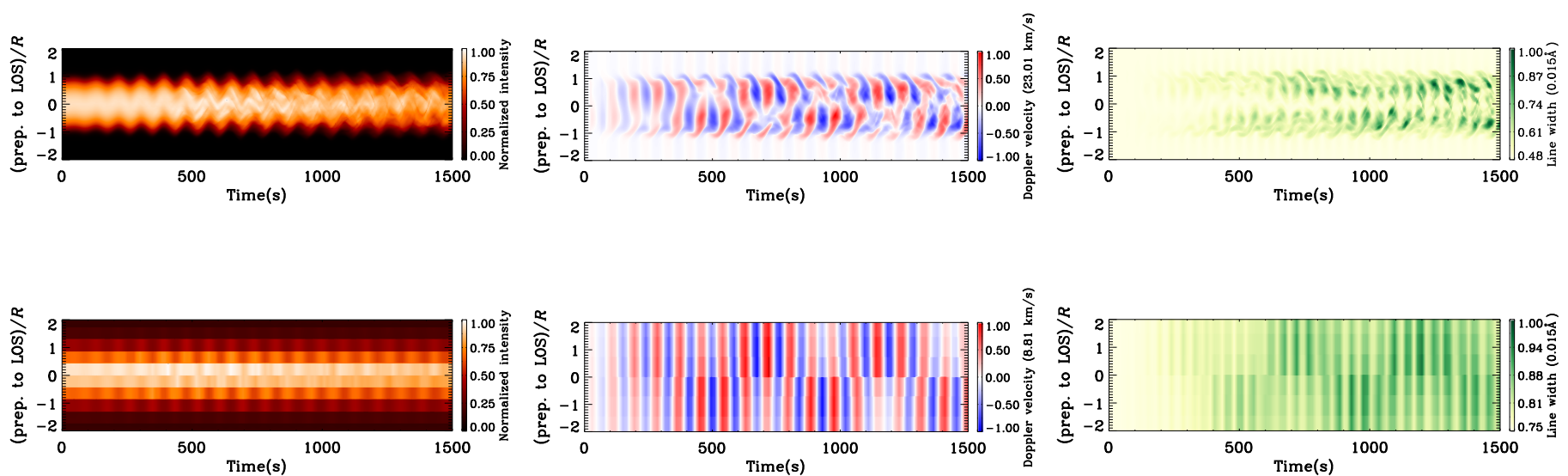

Figure 7. Forward-modeling results for the three models in the Fe IX $171 \AA$ line at the apex with an LOS angle of $45^{\circ}$. Left panel of each model: time-distance maps of the normalized intensities. The upper one is obtained with the full numerical resolution and the lower one with a degraded resolution comparable to SDO/AIA. Middle panel of each model: time-distance maps of the normalized Doppler velocity. The upper one is obtained with the full numerical resolution and the lower one with a degraded resolution comparable to Hinode/EIS. Right panel of each model: similar to the Doppler velocity maps, but for the spectral line width.

use a spectral resolution of $36 \mathrm{~km} \mathrm{~s}^{-1}$ and a temporal resolution of $15 \mathrm{~s}$ to mimic Hinode/EIS. The bottom row of the middle panel of Figure 7(a) shows that the "bow-like" shapes cannot be detected any more, due to the limited resolution. The shapes become staggered red and blue stripes. The right column of Figure 7 (a) is similar to the middle panel, but for the spectral line width. We cannot see the obvious line broadening before about $t=200 \mathrm{~s}$. This is because the initially formed oscillations in our loop have relatively small amplitudes, causing indistinguishable broadening. Then, the small structures generate rapidly around the loop boundary, showing a significant broadening. Similarly, the degraded results 
mimicking Hinode/EIS are shown in the bottom row of the right panel of Figure 7(a). The fine structures cannot be seen; only stripes are detectable.

Figure 7(b) shows the forward model of the A-model. The original and degraded resolution results of the imaging models can be seen in the left panel of Figure 7(b). No transverse oscillations appear, largely because the azimuthal incompressible Alfvén modes do not disturb density. But intensity fluctuations can be seen in the original resolution results after $500 \mathrm{~s}$, due to the KH instability induced through phase mixing. The fluctuations are not visible in the degraded resolution. Therefore, the imaging instruments cannot observe torsional Alfvén modes at their current resolution. The middle panel of Figure 7(b) shows the Doppler velocity maps. The original resolution results (upper row) present staggered spot regions, showing that axisymmetric Alfvén oscillations are set up in the loop. Similar to the K-model case, staggered red and blue stripes appear in the degraded models. We can also find signatures of Alfvén oscillations in the right panel of Figure 7(b), where the line width broadens inside the loop owing to the torsional motions. Only stripes are detectable in the bottom row of the right panel of Figure 7(b) because of the coarse resolution.

Figure 7(c) shows the forward-modeled results of the M-model. Fine structures can be seen in the intensity diagram as for the K-model. After about $800 \mathrm{~s}$, the structures seem more disordered, owing to the torsional motions of Alfvén modes. The same degradation procedure is done to mimic the observations of $S D O /$ AIA. Due to the limited resolution, neither the Alfvén properties nor fine structures can be observed. This diagram is similar to the K-model case, and they are both very similar to the real observations, meaning that both models could provide explanations for the decayless oscillations. The Doppler velocity map here also presents the blue- and redshifts, but showing "tadpole-like" shapes. The torsional Alfvén waves break the "bows" into smaller "tadpole" pairs. Due to the rotating and transverse motions, superpositions happen at the "heads" and cancellations happen at the regions with no "tadpole." In the bottom row of the middle panel of Figure 7(c), with the resolution of Hinode/EIS, the "tadpole-like" shapes cannot be detected either; red and blue stripes are generated instead. The right column of Figure 7(c) shows the line width maps. As mentioned above, the mixed wave modes can induce more turbulent structures. Therefore, the line broadening can be observed in almost the whole loop region, and disordered broadening shapes can be seen. Similar to the Doppler shift properties, the fine structures in line width cannot be observed in the lower row of the right panel of Figure 7(c). Considering the frequency mismatch between the kink modes and Alfvén modes in the M-model, we would expect a beating behavior between these two wave modes. As is shown in Figure 7(c), the increases in Doppler velocity and line width show beatings, which can also be observed in the modulation of the kinetic and magnetic energy amplitudes in Figure 5(c).

We plot the oscillation profiles of the degraded resolution intensities, as is shown in Figure 8. The intensity profiles are the maximum values of Gaussian fits of the results in the bottom left of Figures 7(a) and (c). The profiles of these two models do not have significant difference, indicating that only kink period signals can be observed by $S D O /$ AIA. The amplitude here is about $0.1 \mathrm{Mm}$, which agrees with the observed values in Anfinogentov et al. (2013).

We note that the staggered pattern of Doppler velocity in the A-model sets a clear difference with the case in the K-model. Actually, this has not been detected yet with EIS. It indicates that the amplitudes of torsional Alfvén waves assumed inside the loop are probably larger than the real ones. Besides, the more localized distribution of the torsional Alfvén modes would also influence the Doppler velocity in the coarse resolution case. The more localized the distribution is, the smaller Doppler velocity we can obtain when degrading the full numerical resolution. On top of that, if we keep the same annular velocity shape but allow the same amplitude over a broader region that includes the boundary layer, we would have a strong superposition of Alfvén waves with different periods, leading to very weak signals in a spectrograph. This is actually suggested in Antolin et al. (2018) in order to explain some spicules observations. However, neither an adjustment of amplitude nor a more localized Alfvén driver model will influence our previous statement that the KHI eddies can help to efficiently dissipate the energy in other wave modes.

Therefore, we can distinguish Alfvén modes and kink modes through the properties of fine structures in imaging models and particular shapes of the Doppler velocity and line width properties in spectral models with the original numerical resolution. Neither small structures nor particular shapes can be observed owing to the limitation of the resolving power of real instruments. Hence, Alfvén modes can probably coexist with kink modes, leading to enhanced heating, while being hidden from imaging instruments. This means that the ubiquitous decayless oscillations in coronal loops can play an important role in coronal heating by the enhanced heating of unresolved modes.

\section{Discussions and Conclusions}

In this study, we simulated different oscillations in coronal loops, using a kink driver, an Alfvén driver, and a mixed Alfvén and kink driver located at the footpoints of flux tubes. For all models, the oscillations excited in loops can lead to the $\mathrm{KHI}$ and generate small eddies. Especially in the M-model, the torsional motions together with transverse motions can help to generate more eddies. Besides, the Alfvén oscillations coming from the driver inside the loop and from kink oscillations due to resonant absorption will have phase mixing, which further enhanced the instability.

We can indeed observe the increase of internal energy and temperature. The heating is enhanced for the simulation containing the mixed driver, compared with the other two models. This means that the mixed modes can lead to a more efficient energy dissipation in the turbulent state of plasma and that the KHI acts as an agent to dissipate wave energy in other modes.

According to Heyvaerts \& Priest (1983), the KHI vortices can also be induced by phase-mixed standing Alfvén modes. In turn, the small vortices can also reinforce the phase mixing. This process makes more and more fine structures, which can help to dissipate wave energy more efficiently. However, in our simulations, the smaller and smaller scales will become close to the spatial numerical resolution eventually, and we cannot always observe the finest structures generated in loops. Generally, if we can capture smaller fine structures, the heating effects could be more pronounced. 


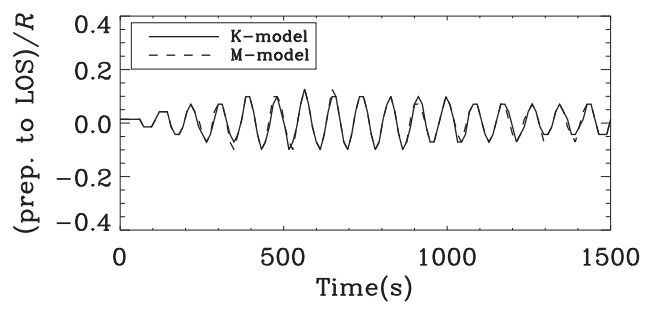

Figure 8. Oscillation profiles of degraded resolution intensities, obtained by calculating the maximum values of Gaussian fits of the results in the bottom left rows of Figures 7(a) and (c). Solid and dashed lines represent the results of the $\mathrm{K}$-model and the M-model, respectively.

Forward models can help to compare to the observations. Fine structures can be observed in the obtained imaging models. However, neither Alfvén modes nor small structures are observable in the degraded resolution models. As such, the obtained imaging models agree with the decayless oscillations detected through SDO/AIA. Therefore, this study shows that Alfvén waves can probably coexist with transverse waves in coronal loops, leading to enhanced heating. Our spectral models reveal fine structures, the Doppler shift, and the line width properties. Neither fine structures nor the particular properties can be observed in the coarse resolution models mimicking Hinode/EIS. However, beatings can be observed in Doppler velocity and line width in the mixed driver model

We notice that in the near future a new generation of highresolution ground-based instruments, such as the Daniel K. Inouye Solar Telescope (DKIST)/Diffraction Limited Near Infrared Spectropolarimeter (DL-NIRSP), will help to detect more detailed structures and reveal the energy release processes in the solar corona. The potential of this instrument has been recently predicted by Snow et al. (2018). The highest spatial sampling size of the forthcoming DKIST/DL-NIRSP is 0 ". 03 , which is suitable for disk and limb observations, while the wide-field mode with a spatial resolution of 0 ". 464 will provide coronal observations. Within the temperature range in our current models ( $\sim 1 \mathrm{MK})$, DL-NIRSP may have the ability to recognize the fine structures demonstrated in our forward models due to the high resolution. Similarly, forward modeling for next-generation instrumentation targeting the recently proposed MUlti-slit Solar Explorer has been done in Antolin et al. (2017). It is shown that most of the features from the TWIKH rolls in coronal loops can be detected with a spatial resolution of 0 ." 33 and a spectral resolution of $25 \mathrm{~km} \mathrm{~s}^{-1}$. Therefore, the future high-resolution instruments may help to reveal the turbulent motions in coronal loops and distinguish different numerical models.

We assumed a uniform temperature distribution in the whole simulation domain, which can help to recognize the heating effects from waves more clearly. According to Karampelas et al. (2017), the mixing between the colder loop and the hotter corona caused a drop larger than $1.5 \%$ in the averaged temperature, while simulations with a uniform temperature lead to a rise of about $0.25 \%$. This means that the gradient of the temperature can largely hide the expected heating from waves. Once introducing such a temperature gradient, we can hardly expect a noticeable temperature increase as in our results here, even considering the stronger plasma driving in the M-model. The larger magnetic field $(50 \mathrm{G})$ in our models leads to a direct consequence of a smaller transverse oscillation period (87 s). However, this value is still in the scatter range of relatively shorter loop observations reported by Anfinogentov et al. (2015) and Goddard et al. (2016). Meanwhile, the kink speed in our loops is $c_{k} \approx 3452 \mathrm{~km} \mathrm{~s}^{-1}$, which is close to the fitting value of $3300 \mathrm{~km} \mathrm{~s}^{-1}$ in Goddard et al. (2016).

Our models still lack realistic solar atmospheric conditions, such as vertical stratification due to gravity. The vertical nonuniform layer may lead to the reflection of waves, which can probably influence the energy carrying capability of waves and the generation of KHI. The different transverse distributions of parameters, which are usually studied analytically involving different wave modes (Soler et al. 2014; Guo et al. 2016), will influence the resonant absorption and also the phase mixing of Alfvén modes at different magnetic surfaces, thereby influencing the dissipation efficiency. On top of that, the magnetic field variation with height and the loop curvature (Van Doorsselaere et al. 2004; van Doorsselaere et al. 2009) are also neglected in our current models. To clarify their influence on wave heating, we will conduct a series of studies on more realistic curved loops in nonuniform force-free magnetic field in future works.

This project has received funding from the European Research Council (ERC) under the European Union's Horizon 2020 research and innovation program (grant agreement Nos. 724326 and 647214). B.L. is supported by the National Natural Science Foundation of China (41674172, 41474149, and 11761141002). M.G. acknowledges the funding from the China Scholarship Council (CSC) and GOA-2015-014 (KU Leuven). T.V.D. is supported by the IAP P7/08 CHARM (Belspo) and the GOA-2015-014 (KU Leuven). P.A. acknowledges funding from his STFC Ernest Rutherford Fellowship (No. ST/R004285/1).

\section{ORCID iDs}

Mingzhe Guo (iD https://orcid.org/0000-0003-4956-6040 Tom Van Doorsselaere (D) https://orcid.org/0000-00019628-4113

Konstantinos Karampelas (1) https://orcid.org/0000-00015507-1891

Bo Li (iD https://orcid.org/0000-0003-4790-6718

Patrick Antolin (iD https://orcid.org/0000-0003-1529-4681

Ineke De Moortel (i) https://orcid.org/0000-0002-1452-9330

\section{References}

Anfinogentov, S., Nisticò, G., \& Nakariakov, V. M. 2013, A\&A, 560, A107 Anfinogentov, S. A., Nakariakov, V. M., \& Nisticò, G. 2015, A\&A, 583, A136 Antolin, P., De Moortel, I., Van Doorsselaere, T., \& Yokoyama, T. 2016, ApJL, 830, L22

Antolin, P., De Moortel, I., Van Doorsselaere, T., \& Yokoyama, T. 2017, ApJ, 836,219

Antolin, P., Schmit, D., Pereira, T. M. D., De Pontieu, B., \& De Moortel, I. 2018, ApJ, 856, 44

Antolin, P., Yokoyama, T., \& Van Doorsselaere, T. 2014, ApJL, 787, L22

Arregui, I., Oliver, R., \& Ballester, J. L. 2012, LRSP, 9, 2

Aschwanden, M. J. 2006, RSPTA, 364, 417

Aschwanden, M. J., de Pontieu, B., Schrijver, C. J., \& Title, A. M. 2002, SoPh, 206, 99

Aschwanden, M. J., Fletcher, L., Schrijver, C. J., \& Alexander, D. 1999, ApJ, 520,880

Aschwanden, M. J., Nightingale, R. W., Andries, J., Goossens, M., \& Van Doorsselaere, T. 2003, ApJ, 598, 1375

Beliën, A. J. C., Martens, P. C. H., \& Keppens, R. 1999, ApJ, 526, 478

Cally, P. S., \& Moradi, H. 2013, MNRAS, 435, 2589

Chen, Y., Feng, S. W., Li, B., et al. 2011, ApJ, 728, 147

Chen, Y., Song, H. Q., Li, B., et al. 2010, ApJ, 714, 644

De Moortel, I., \& Nakariakov, V. M. 2012, RSPTA, 370, 3193

De Pontieu, B., McIntosh, S. W., Carlsson, M., et al. 2007, Sci, 318, 1574

Edwin, P. M., \& Roberts, B. 1983, SoPh, 88, 179 
Goddard, C. R., Nisticò, G., Nakariakov, V. M., \& Zimovets, I. V. 2016, A\&A, 585, A137

Goossens, M., Andries, J., \& Aschwanden, M. J. 2002, A\&A, 394, L39

Goossens, M., Hollweg, J. V., \& Sakurai, T. 1992, SoPh, 138, 233

Grant, S. D. T., Jess, D. B., Zaqarashvili, T. V., et al. 2018, NatPh, 14, 480

Guo, M.-Z., Chen, S.-X., Li, B., Xia, L.-D., \& Yu, H. 2016, SoPh, 291, 877

Gupta, G. R., Banerjee, D., Teriaca, L., Imada, S., \& Solanki, S. 2010, ApJ, 718,11

He, J., Marsch, E., Tu, C., \& Tian, H. 2009, ApJL, 705, L217

Heyvaerts, J., \& Priest, E. R. 1983, A\&A, 117, 220

Hollweg, J. V., \& Yang, G. 1988, JGR, 93, 5423

Howson, T. A., De Moortel, I., \& Antolin, P. 2017a, A\&A, 602, A74

Howson, T. A., De Moortel, I., \& Antolin, P. 2017b, A\&A, 607, A77

Jess, D. B., Mathioudakis, M., Erdélyi, R., et al. 2009, Sci, 323, 1582

Jess, D. B., Morton, R. J., Verth, G., et al. 2015, SSRv, 190, 103

Jess, D. B., Reznikova, V. E., Ryans, R. S. I., et al. 2016, NatPh, 12, 179

Kaneko, T., Goossens, M., Soler, R., et al. 2015, ApJ, 812, 121

Karampelas, K., \& Van Doorsselaere, T. 2018, A\&A, 610, L9

Karampelas, K., Van Doorsselaere, T., \& Antolin, P. 2017, A\&A, 604, A130

Khomenko, E., \& Cally, P. S. 2012, ApJ, 746, 68

Kuridze, D., Morton, R. J., Erdélyi, R., et al. 2012, ApJ, 750, 51

Kuridze, D., Verth, G., Mathioudakis, M., et al. 2013, ApJ, 779, 82

Kwon, R.-Y., Ofman, L., Olmedo, O., et al. 2013, ApJ, 766, 55

Magyar, N., Van Doorsselaere, T., \& Marcu, A. 2015, A\&A, 582, A117

Mann, I. R., Wright, A. N., \& Cally, P. S. 1995, JGR, 100, 19441

Matsumoto, T., \& Shibata, K. 2010, ApJ, 710, 1857

Mignone, A., Bodo, G., Massaglia, S., et al. 2007, ApJS, 170, 228

Morton, R. J., Verth, G., Jess, D. B., et al. 2012, NatCo, 3, 1315

Muller, R., Roudier, T., Vigneau, J., \& Auffret, H. 1994, A\&A, 283, 232

Nakariakov, V. M., Anfinogentov, S. A., Nisticò, G., \& Lee, D.-H. 2016, A\&A, 591, L5

Nakariakov, V. M., \& Ofman, L. 2001, A\&A, 372, L53

Nakariakov, V. M., Ofman, L., Deluca, E. E., Roberts, B., \& Davila, J. M. 1999, Sci, 285, 862
Nakariakov, V. M., Pilipenko, V., Heilig, B., et al. 2016, SSRv, 200, 75

Nakariakov, V. M., \& Verwichte, E. 2005, LRSP, 2, 3

Nisticò, G., Nakariakov, V. M., \& Verwichte, E. 2013, A\&A, 552, A57

Ofman, L., Davila, J. M., \& Steinolfson, R. S. 1994, ApJ, 421, 360

Ofman, L., Klimchuk, J. A., \& Davila, J. M. 1998, ApJ, 493, 474

Pagano, P., Pascoe, D. J., \& De Moortel, I. 2018, A\&A, 616, A125

Parnell, C. E., \& De Moortel, I. 2012, RSPTA, 370, 3217

Pascoe, D. J., Wright, A. N., \& De Moortel, I. 2010, ApJ, 711, 990

Raes, J. O., Van Doorsselaere, T., Baes, M., \& Wright, A. N. 2017, A\&A, 602, A75

Ruderman, M. S., \& Erdélyi, R. 2009, SSRv, 149, 199

Schrijver, C. J., Title, A. M., Berger, T. E., et al. 1999, SoPh, 187, 261

Snow, B., Botha, G. J. J., Scullion, E., et al. 2018, ApJ, 863, 172

Soler, R., Goossens, M., Terradas, J., \& Oliver, R. 2014, ApJ, 781, 111

Soler, R., \& Terradas, J. 2015, ApJ, 803, 43

Taroyan, Y., \& Erdélyi, R. 2009, SSRv, 149, 229

Terradas, J., Andries, J., Goossens, M., et al. 2008, ApJL, 687, L115

Tomczyk, S., \& McIntosh, S. W. 2009, ApJ, 697, 1384

Tomczyk, S., McIntosh, S. W., Keil, S. L., et al. 2007, Sci, 317, 1192

Van Doorsselaere, T., Antolin, P., Yuan, D., Reznikova, V., \& Magyar, N. 2016, FrASS, 3, 4

Van Doorsselaere, T., Debosscher, A., Andries, J., \& Poedts, S. 2004, A\&A, 424, 1065

Van Doorsselaere, T., Nakariakov, V. M., \& Verwichte, E. 2007, A\&A, 473, 959

Van Doorsselaere, T., Nakariakov, V. M., Young, P. R., \& Verwichte, E. 2008, A\&A, 487, L17

van Doorsselaere, T., Verwichte, E., \& Terradas, J. 2009, SSRv, 149, 299

Verwichte, E., Nakariakov, V. M., \& Cooper, F. C. 2005, A\&A, 430, L65

Vranjes, J., Poedts, S., Pandey, B. P., \& de Pontieu, B. 2008, A\&A, 478, 553

Wentzel, D. G. 1979, A\&A, 76, 20

Withbroe, G. L., \& Noyes, R. W. 1977, ARA\&A, 15, 363

Zajtsev, V. V., \& Stepanov, A. V. 1975, IGAFS, 37, 3

Zaqarashvili, T. V. 2003, A\&A, 399, L15 